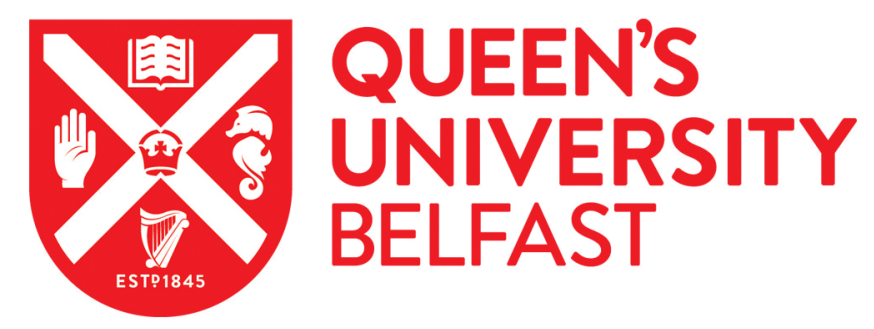

\title{
Melanism and disease resistance in insects
}

Wilson, K., Cotter, S., Reeson, A. F., \& Pell, J. K. (2001). Melanism and disease resistance in insects. Ecology Letters, 4(6), 637-649.

\author{
Published in: \\ Ecology Letters
}

Document Version:

Publisher's PDF, also known as Version of record

Queen's University Belfast - Research Portal:

Link to publication record in Queen's University Belfast Research Portal

\footnotetext{
General rights

Copyright for the publications made accessible via the Queen's University Belfast Research Portal is retained by the author(s) and / or other copyright owners and it is a condition of accessing these publications that users recognise and abide by the legal requirements associated with these rights.

Take down policy

The Research Portal is Queen's institutional repository that provides access to Queen's research output. Every effort has been made to ensure that content in the Research Portal does not infringe any person's rights, or applicable UK laws. If you discover content in the Research Portal that you believe breaches copyright or violates any law, please contact openaccess@qub.ac.uk.
} 


\section{REPORT}

\section{Melanism and disease resistance in insects}

Kenneth Wilson, ${ }^{1 *}$ Sheena C. Cotter, ${ }^{1}$ Andrew F. Reeson ${ }^{2}$ and Judith K. Pell ${ }^{3}$

${ }^{1}$ Institute of Biological Sciences, University of Stirling, Stirling,

FK9 4LA, UK.

${ }^{2}$ Applied \& Molecular Ecology,

Adelaide University, Waite

Campus, Glen Osmond 5064,

South Australia.

${ }^{3}$ Plant and Invertebrate Ecology

Department, IACR -

Rothamsted, Harpenden, Herts

AL5 2JQ, UK.

*Correspondence: E-mail:

ken.wilson@stirling.ac.uk

\begin{abstract}
There is growing evidence that insects in high-density populations invest relatively more in pathogen resistance than those in low-density populations (i.e. density-dependent prophylaxis). Such increases in resistance are often accompanied by cuticular melanism, which is characteristic of the high-density form of many phase polyphenic insects. Both melanism and pathogen resistance involve the prophenoloxidase enzyme system. In this paper the link between resistance, melanism and phenoloxidase activity is examined in Spodoptera larvae. In $S$. exempta, cuticular melanism was positively correlated with phenoloxidase activity in the cuticle, haemolymph and midgut. Melanic S. exempta larvae were found to melanize a greater proportion of eggs of the ectoparasitoid Euplectrus laphygmae than non-melanic larvae, and melanic S. littoralis were more resistant to the entomopathogenic fungus Beauveria bassiana (in S. exempta the association between melanism and fungal resistance was non-signficant). These results strengthen the link between melanism and disease resistance and implicate the involvement of phenoloxidase.
\end{abstract}

\section{Keywords}

Density-dependent prophylaxis, fungal pathogen, melanism, parasitoid, phase polyphenism, phenoloxidase, phenotypic plasticity, resistance.

Ecology Letters (2001) 4: 637-649

\section{INTRODUCTION}

Density-dependent prophylaxis (DDP) is the phenomenon in which individuals invest more in immune function when at high population densities, as a counter-measure to density-dependent pathogen transmission rates (Wilson \& Reeson 1998). Evidence in support of DDP is accruing from a number of insects that exhibit density-dependent phase polyphenism (see Long 1953; Mitsui \& Kunimi 1988; Kunimi \& Yamada 1990; Goulson \& Cory 1995; Reeson et al. 1998; Barnes \& Siva-Jothy 2000; Reeson et al. 2000). In all of these cases, larval crowding is associated with cuticular melanization ('melanism'; Fig. 1a). The co-occurrence of melanism and increased levels of disease resistance in the high-density form of phase polyphenic insects raises the possibility that the two phenomena are closely linked.

Five studies have examined the relationship between melanism and disease resistance whilst controlling for larval density. Melanic Spodoptera exempta larvae were up to four times more resistant to a nucleopolyhedrovirus (NPV) than non-melanic conspecifics (Reeson et al. 1998), melanic Mythimna separata larvae were twice as resistant to NPV (Kunimi \& Yamada 1990) and five times more resistant to an entomopathogenic fungus (Mitsui \& Kunimi 1988), and melanic Tenebrio molitor were up to three times more resistant to an entomopathogenic fungus (Barnes \& Siva-Jothy 2000). In contrast, melanic Mamestra brassicae larvae were significantly more susceptible to NPV than non-melanics (Goulson \& Cory 1995). Thus, in four out of five studies in which density-dependent effects have been controlled for, there was a positive association between melanism and pathogen resistance, and a number of authors have suggested that melanism may be a useful marker for high levels of investment in immune defence in insects (Majerus 1998; Reeson et al. 1998; Barnes \& Siva-Jothy 2000). If such an association exists, what is the mechanism generating it? In this paper, we argue that the cuticular melanin is functional in disease resistance and is one of a suite of prophylactic resistance traits exhibited by insects in response to the increased threat of disease associated with crowding.

The first, and probably best, lines of defence against most parasites and pathogens are the cuticle and the midgut. Thus, any modifications that enhance their ability to act as physical or chemical barriers to penetration by entomopathogens are likely to be favoured when the threat of disease is high (e.g. during periods of crowding). Melanin has at least two properties that are likely to increase immunocompetence (sensu Owens \& Wilson 1999). First, because it is a polymer, melanin is likely to strengthen the cuticle and so improve its ability to act as a physical barrier 

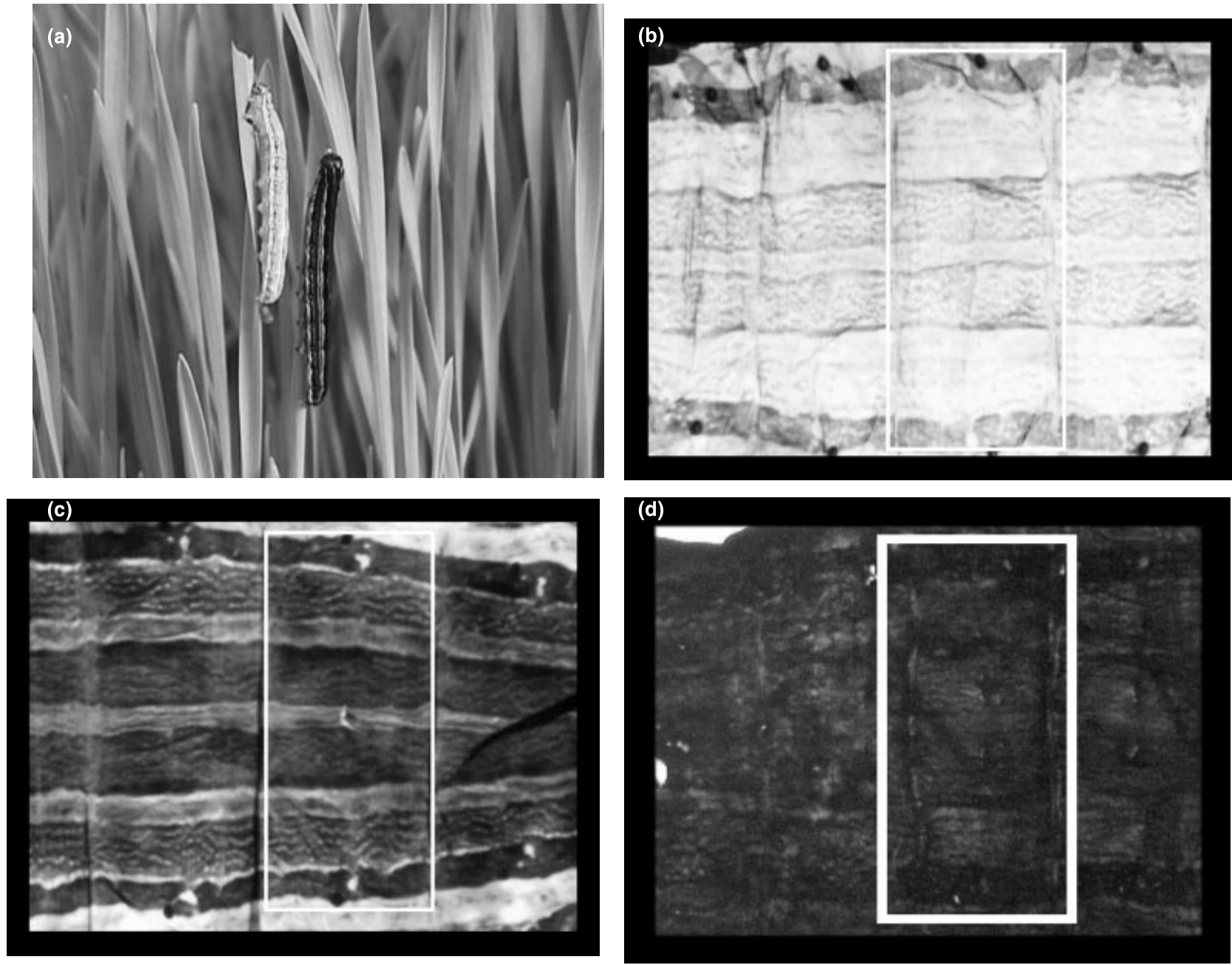

Figure 1 Density-dependent cuticular melanization in Spodoptera exempta. (a) Live larvae, showing the pale, low-density phenotype on the left and the dark, high-density phenotype on the right; (b) the dorsal cuticle of the pale phenotype; (c) the dorsal cuticle of the dark phenotype; (d) the dorsal cuticle of the pale phenotype following incubation in L-dopa. Cuticular melanization was measured as the mean and maximum density score in the area shown by the white boxes in (b)-(d).

to the penetration of cutaneously entering parasites and pathogens, such as fungi, bacteria and even parasitoids (St. Leger et al. 1988; Hajek \& St. Leger 1994). Second, and perhaps more importantly, melanin is toxic to microorganisms and has potent antimicrobial activity (e.g. Montefiori \& Zhou 1991; Ourth \& Renis 1993; Sidibe et al. 1996; Ishikawa et al. 2000). The mechanisms for this are unclear at present, but a number of studies have shown that melanin binds to a range of proteins (e.g. Doering et al. 1999) and inhibits many of the lytic enzymes produced by microorganisms, including proteases and chitinases (Kuo \& Alexander 1967; Bull 1970). Söderhäll \& Ajaxon (1982) showed that when the crayfish-parasitic fungus Aphanomyces astaci was grown on a PG-I agar medium, there was significant inhibition of fungal growth when the growth medium contained melanin or any of its constituent quinones. Moreover, St Leger et al. (1988) showed that when larval cuticles of Manduca sexta were induced to melanize, they resisted fungal penetration for $30 \mathrm{~h}$ longer than unmelanized cuticles. Thus, melanin may enhance disease resistance in insects not only by improving the physical properties of insect cuticle, but also by enhancing its chemical properties.

The prophenoloxidase cascade is a suite of enzymes that oxidize tyrosine derivatives to their corresponding quinones and their polymerization product, melanin (Mason 1955; Hiruma \& Riddiford 1988; Nappi \& Vass 1993). These enzymes are involved not only in cuticular melanization, but also in the various immune responses directed against parasites and pathogens, including cellular encapsulation, humoral encapsulation and nodule formation (Poinar 1974; Götz 1986; Paskewitz et al. 1988; Hung \& Boucias 1992; Beckage et al. 1993). Phenoloxidase production is activated 
in the cuticle when it is punctured or stimulated by microbial cell wall components (including peptidoglycan, $\beta-1,3$ glucan and possibly lipopolysaccaride), resulting in the local production of toxic quinones and melanin in the cuticle, which can reduce fungal growth and immobilize bacteria (St Leger 1991; Marmaras et al. 1993). The midgut is an important site for resisting pathogens that enter the host orally, such as baculoviruses, protozoa and many bacteria, and melanin is also produced during this process, suggesting a role for phenoloxidase (PO). For example, the spread of baculovirus in non-permissive hosts appears to be blocked by aggregations of haemocytes that form melanotic capsules around infected cells in the midgut trachea (Washburn et al. 1996, 2000). Similarly, in a mosquito selected for resistance to malaria, parasite ookinetes are melanized between the midgut epithelial cells and the basal laminae (Collins et al. 1986). These resistant insects also show higher PO activity in the midgut following exposure to the parasite (Paskewitz et al. 1989). Moreover, in tsetse flies (Glossina spp.), there was a significant positive association between PO activity and refractoriness to the protozoan Trypanosoma brucei rhodesiense, both within species (male vs. female G. morsitans morsitans) and among species (G. m. morsitans vs. G. palpalis palpalis) (Nigam et al. 1997).

If melanin enhances resistance to parasites and pathogens, both when it is a static component of the insect cuticle and when it is produced de novo in response to pathogen attack (via the action of the prophenoloxidase cascade), then both cuticular melanization and enhanced phenoloxidase activity might be expected to increase in response to cues predicting likely disease threat, such as increased population density. So far, the only studies to have examined the link between DDP and PO have done so using PO measured in the haemolymph. In $S$. exempta, haemolymph PO activity was greatest in melanic larvae and in individuals reared under crowded conditions (Reeson et al. 1998), whereas in T. molitor there was no association between haemolymph $\mathrm{PO}$ and rearing density (the correlation between PO and melanism was not determined directly; Barnes \& Siva-Jothy 2000).

The main aim of the present study was therefore to quantify density-dependent production of PO in the key sites for pathogen resistance: the cuticle, haemolymph and midgut. We also set out to determine the relationships between PO levels across these tissues and how these relate to cuticular melanization; do individuals with melanic cuticles have higher levels of PO in the cuticle and are PO levels in the different tissues correlated? Finally, we tested whether density-dependent changes in the cuticle were associated with variation in resistance to parasites and pathogens that access their hosts percutaneously, using an ectoparasitoid and an entomopathogenic fungus. As our model system, we used Lepidopteran larvae from the genus Spodoptera: the African armyworm, S. exempta and the
Egyptian cotton leafworm, S. littoralis. Both of these species exhibit density-dependent phase polyphenism, developing melanized cuticles in response to larval crowding (Faure 1943; Hodjat 1970; Gunn 1998).

\section{MATERIALS AND METHODS}

\section{Rearing of larvae}

Except where stated, all experiments used fourth-instar $S$. exempta larvae from laboratory stocks that had been maintained at $27^{\circ} \mathrm{C}$ and $12: 12$ light : dark photoperiod for at least 10 generations. Larvae were reared from within $48 \mathrm{~h}$ of hatching at either one (solitary-reared) or four (crowd-reared) larvae per $12 \mathrm{~mL}$ plastic pot. During this time they were fed on a wheatgerm-based artificial diet and their colour generally ranged from pale grey to jet black. For the parasitoid experiment, larval colour was scored by eye on a five-point scale: $-2=$ very pale, $-1=$ pale, $0=$ mid, $1=$ dark, 2 = very dark, but in the other experiments, we categorized larvae as simply pale (score $=-2$ to 0 ) and dark (score $=1$ or 2 ). Using image analysis software, we were subsequently able to quantify the difference between pale and dark cuticles (see below).

In phase polyphenic species, including $S$. exempta, development into the dark, high-density (gregaria) phenotype is triggered by the perception of (usually tactile) cues typical of high population densities (e.g. Kazimirova 1992). However, the threshold density at which this phenotypic change occurs is under genetic control (e.g. Goulson 1994), and so even when reared solitarily the development of some individuals is stimulated towards that of the high-density phenotype. Thus, we can consider these 'dark-solitary' larvae to be at an intermediate position on the gregarization scale (i.e. they exhibit traits that are intermediate between the typical solitaria and gregaria phenotypes). As 'pale-crowded' larvae are rare in S. exempta, we were generally able to assess the colour of only three phenotypes: the 'typical' pale-solitary form (Fig. 1a, left), the 'typical' dark-crowded form (Fig. 1a, right), and the dark-solitary form, which looks like the typical crowded form to the naked eye (in the statistical analyses below, these are referred to as phenotypes 1, 3 and 2, respectively).

Spodoptera littoralis had been in culture for eight generations at the start of this study. The rearing conditions were the same as for $S$. exempta, except that all larvae were reared at $25{ }^{\circ} \mathrm{C}$ and crowded larvae were produced at a density of three larvae per $12 \mathrm{~mL}$ pot.

\section{Phenoloxidase assays}

Haemolymph was extracted from each larva between the last pair of prolegs, and $8 \mu \mathrm{L}$ was placed in $400 \mu \mathrm{L}$ of ice-cold phosphate-buffered saline (PBS, pH 7.4; Sambrook 
et al. 1989) and mixed in a plastic Eppendorf tube. The sample was frozen to disrupt haemocyte membranes and PO activity in the defrosted sample was assayed spectrophotometrically using L-dopa as a substrate (after Ashida \& Söderhäll 1984). This involved pipetting triplicate $100 \mu \mathrm{L}$ samples of the buffered haemolymph into a microtitre plate, adding $100 \mu \mathrm{L}$ of $20 \mathrm{~mm} \mathrm{~L}$-dopa to each and incubating the mixture at $25{ }^{\circ} \mathrm{C}$. The absorbance was read at $492 \mathrm{~nm}$ on a temperature-controlled VERSAMAX tunable microplate reader (Molecular Devices Corporation, Sunnyvale, CA) after $20 \mathrm{~min}$, which was during the linear phase of the reaction. Using $10 \mu \mathrm{L}$ of the haemolymph/PBS mixture, the amount of protein in the sample was also measured (calibrated using a standard curve created on the same microtitre plate using a BSA standard). Phenoloxidase activity is expressed as $\mathrm{PO}$ units per $\mathrm{mg}$ protein, where one unit is the amount of enzyme required to increase the absorbance by $0.001 \mathrm{~min}^{-1}$.

Following haemolymph extraction, the midguts and dorsal cuticles were dissected from each larva and fixed for $1 \mathrm{~h}$ in $2 \%$ formaldehyde and $0.5 \%$ glutaraldehyde in PBS (Wolfgang \& Riddiford 1981). After fixation, the cuticles and midguts were washed over $3 \mathrm{~h}$ in three changes of PBS. They were then cut in half and each piece weighed. One half of each midgut and cuticle was then placed in $1 \mathrm{~mL}$ of $20 \mathrm{~mm}$ L-dopa. The other half was placed in $1 \mathrm{~mL}$ of $20 \mathrm{~mm}$ L-dopa saturated with phenylthiourea (PTU) as a control (PTU acts as a PO inhibitor and none of these samples darkened). After $40 \mathrm{~min}, 200 \mu \mathrm{L}$ of the mixture was pipetted into a microtitre plate and the absorbance measured at $492 \mathrm{~nm}$. PO was expressed as PO units per $\mathrm{g}$ of tissue. Samples of $20 \mathrm{~mm}$ L-dopa alone were incubated along with the experimental samples to act as a control for any natural darkening of the substrate during the experiment. None of these samples darkened, indicating that melanization of all samples was due to endogenous PO.

\section{Cuticular melanization scoring}

Fixed cuticles and midguts were mounted and photographed using a Polaroid DMC digital camera and the images scored for their degree of melanization using Image Pro Plus software (Media Cybernetics 1999). Cuticular melanization was measured as the mean and maximum melanization scores $(1 /$ mean density and $1 /$ min density, respectively, on the Measurements tab of Image Pro Plus). The midgut is fairly homogeneous in colour and so a central area was chosen for scoring. The dorsal cuticle, however, comprises a series of longitudinal stripes varying in their degree of melanization. Thus, mean and maximum melanization was scored for an area that spanned these different degrees of melanization (shown by the boxes in Fig. 1b-d). We also measured melanization in each of the longitudinal stripes.
However, as none of these measures distinguished between pale and dark caterpillars any better than mean density and maximum density, they will not be discussed further.

\section{Resistance to ectoparasitoids}

Six days post-hatch, the colour of solitary-reared and crowdreared larvae was scored and they were left singly overnight with a mated, honeywater-fed Euplectrus laphygmae female in a Petri dish. The following day, the number of eggs laid on the larva was counted. Larvae were maintained singly in labelled pots containing artificial diet. Most eggs hatched within $2-3$ days, but a significant percentage of eggs $(34.7 \pm 4.2 \%$ eggs; mean $\pm \mathrm{SE}, n=59$ larvae) became melanized and failed to develop further. Most of these eggs subsequently appeared to shrink in size and in some cases disappeared, to be replaced by a patch of melanin on the surface of the cuticle. The proportion of parasitoid eggs that became melanized was used as our measure of host resistance to ectoparasitoids. The parasitoids and the larvae used in this experiment were collected in Arusha, Tanzania, in April 1999 and had been in culture for just two generations at the start of the experiment.

\section{Resistance to entomopathogenic fungi}

We assessed resistance to entomopathogenic fungi using an isolate of Beauveria bassiana (reisolation of Mycotrol ${ }^{\circledR}$ strain GHA). The fungus was grown on Sabouraud dextrose agar (SDA) in $9 \mathrm{~cm}$ Petri dishes for 21 days at $23{ }^{\circ} \mathrm{C}$ in darkness. Conidia were harvested from the dishes by scraping into $0.03 \%$ sterile Tween 80 using a sterile scalpel. After filtration through several layers of nylon netting, this provided a stock suspension $\left(1.31 \times 10^{9}\right.$ conidia $\left.\mathrm{ml}^{-1}\right)$ that was diluted to

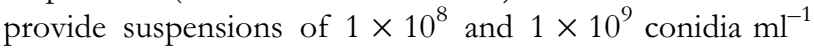
for the bioassay. Control insects were bioassayed with the carrier surfactant $(0.03 \%$ Tween 80$)$. Prior to the bioassay, a sample of conidia from suspension was plated onto SDA and viability was assessed after $24 \mathrm{~h}$ at $23{ }^{\circ} \mathrm{C}$ in darkness. This showed that the percentage germination was $98.3 \%$.

Fungal resistance was assessed in two species of Spodoptera that differed in their susceptibilities to B. bassiana: S. littoralis (the more resistant species at the doses used) and S. exempta (the more susceptible). Solitary-reared and crowd-reared larvae were scored as either pale or dark, and dipped in groups of 12, into $8 \mathrm{~mL}$ of conidial suspension (or Tween control). Inoculated larvae were then placed into a Buchner funnel and the residual suspension removed by vacuum filtration. After inoculation, all individuals were transferred to pots of fresh artificial diet and maintained singly in an incubator at $23{ }^{\circ} \mathrm{C}(\mathrm{L}: \mathrm{D} 12: 12)$. Mortality was recorded daily from 4 days post-inoculation and the cause of mortality was verified by checking for sporulation in cadavers placed in square Petri dishes lined with $1 \%$ water agar. 


\section{Statistical analysis}

All analyses were conducted using either the S-Plus 2000 statistical package (Mathsoft, Inc., Seattle, WA) or Minitab, version 13.1 (Minitab, Inc., PA). Because many of the analyses involved multiple, correlated measurements taken from the same insects (e.g. several measurements of melanism or PO activity), where appropriate, multivariate analysis of variance (MANOVA) was used to analyse the data. This allows one to consider multiple responses as a single multivariate response, rather than a collection of univariate responses, so allowing the covariation between multiple measurements to be explicitly modelled and the probability of Type I errors to be minimized. The statistical significance of the MANOVAs reported here was determined by using Wilks' Lambda $(\Lambda)$, although the alternative tests (e.g. Pillai's trace) always gave similar results. We also report an approximate $F$ and its associated $P$-value, based on a transformation of $\Lambda$. When the MANOva was statistically significant $\left(\mathrm{H}_{0}\right.$ was rejected), univariate ANOVAS were performed for each response variable using sums of squares adjusted for the other dependent variables in the model. Partial correlation coefficients were calculated using the VassarStats website (http://departments.vassar.edu/ lowry/par.html).

\section{RESULTS}

\section{Larval density, colour and melanization of the cuticle and midgut}

Mean and maximum melanization scores were highly correlated (Table 1). Therefore, the relationship between cuticular melanization and other larval attributes was determined using MANOVAs that included mean and maximum melanization scores as dependent variables. The intensity of cuticular melanization was significantly greater in crowd-reared larvae than in larvae reared solitarily (MANOVA: Wilks' lambda, $\Lambda=0.477$, approximate $F_{2,57}=$ 31.28, $P<0.001$; univariate tests: mean: $F_{1,58}=60.50$, $P<0.001$; maximum: $\left.F_{1,58}=47.67, P<0.001\right)$. Thus, both mean and maximum cuticular melanization scores responded independently to changes in larval rearing density, although the mean value appears marginally more responsive. Not surprisingly, within the solitary-reared larvae, dark individuals had significantly higher cuticular melanization scores than pale ones (Wilks' $\Lambda=0.764$, $F_{2,37}=5.70, P=0.007$; univariate tests: mean: $F_{1,38}=$ 10.81, $P=0.002$; maximum: $F_{1,38}=8.04, P=0.007$ ). As a consequence of the above patterns, there was a significant positive relationship between larval phenotype (scored 1-3) and the degree of cuticular melanization (MANOVA: Wilks' $\Lambda=0.394, \quad F_{2,57}=43.91, \quad P<0.001 ; \quad$ univariate tests: mean: $F_{1,58}=83.66, P<0.001$; maximum: $F_{1,58}=64.22$, $P<0.001$; Fig. 2a).

Midgut melanization also varied significantly with rearing density (Wilks' $\Lambda=0.864, F_{2,49}=3.86, P=0.028$; univariate tests: mean: $F_{1,50}=0.01, \quad P>0.9$; maximum: $\left.F_{1,58}=6.42, P=0.014\right)$, but the response was largely restricted to changes in the maximum melanization score. Within solitary-reared larvae, pale and dark individuals did not differ in their degree of midgut melanization (Wilks' $\left.\Lambda=0.919, F_{2,32}=1.41, P>0.2\right)$. Thus, the significant positive relationship between larval phenotype (1-3) and degree of midgut melanization (Wilks' $\Lambda=0.862, F_{2,49}=$ 3.90, $P=0.027$; univariate tests: mean: $F_{1,50}=0.37$,

Table 1 Correlations between phenoloxidase activity (PO) and the degree of cuticular melanization. Pearson's correlation coefficients are shown $(n=60)$. Mean and maximum melanization scores were determined by digital image analysis (see text for details).

\begin{tabular}{|c|c|c|c|c|c|}
\hline & $\begin{array}{l}\text { Haemolymph } \\
\text { PO }\end{array}$ & $\begin{array}{l}\text { Cuticle } \\
\text { PO }\end{array}$ & $\begin{array}{l}\text { Midgut } \\
\text { PO }\end{array}$ & $\begin{array}{l}\text { Mean cuticle } \\
\text { melanization score }\end{array}$ & $\begin{array}{l}\text { Maximum cuticle } \\
\text { melanization score }\end{array}$ \\
\hline Haemolymph PO & & $\begin{array}{l}r=0.480 \\
P<0.001 \\
* * *\end{array}$ & $\begin{array}{l}r=0.421 \\
P<0.001 \\
* * *\end{array}$ & $\begin{array}{l}r=0.408 \\
P=0.002 \\
* *\end{array}$ & $\begin{array}{l}r=0.386 \\
P=0.003 \\
* *\end{array}$ \\
\hline Cuticle PO & & & $\begin{array}{l}r=0.465 \\
P<0.001 \\
* * *\end{array}$ & $\begin{array}{l}r=0.336 \\
P=0.009 \\
* *\end{array}$ & $\begin{array}{l}r=0.230 \\
P=0.077 \\
+\end{array}$ \\
\hline Midgut PO & & & & $\begin{array}{l}r=0.220 \\
P=0.091 \\
+\end{array}$ & $\begin{array}{l}r=0.305 \\
P=0.018 \\
*\end{array}$ \\
\hline Mean cuticle melanization score & & & & & $\begin{array}{l}r=0.847 \\
P<0.001 \\
* * *\end{array}$ \\
\hline
\end{tabular}


(a)

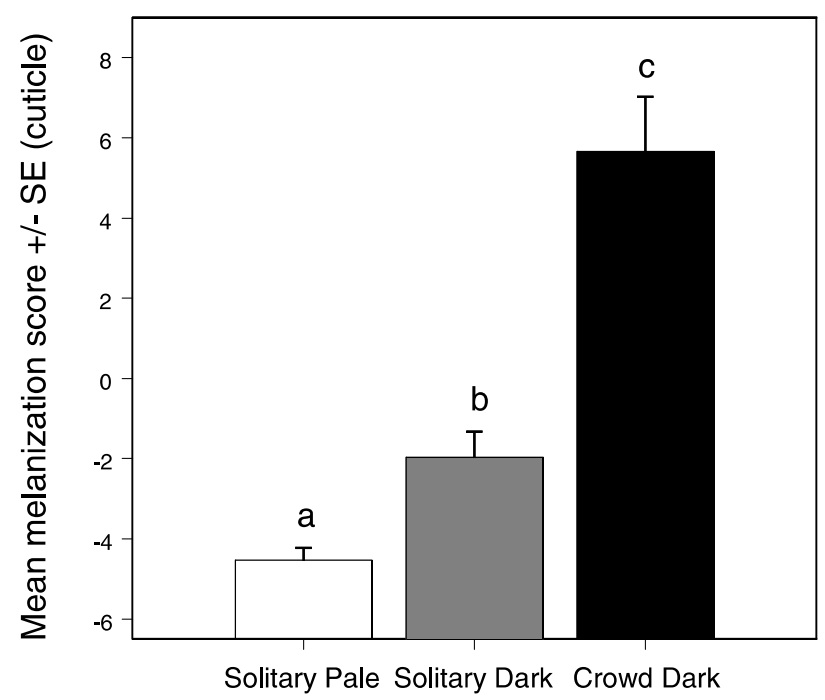

(c)

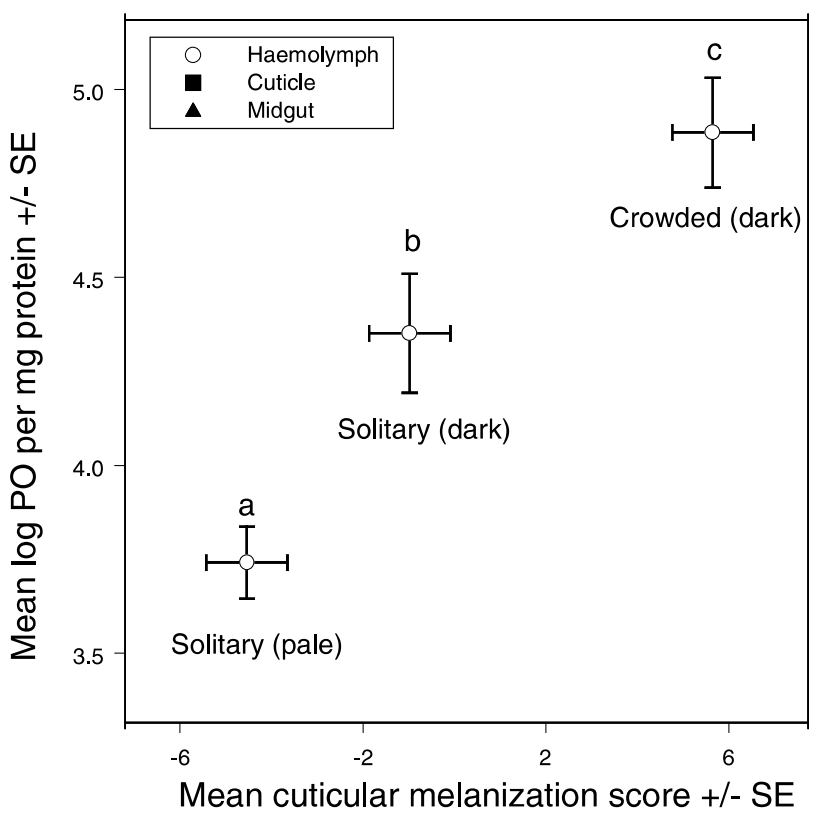

(b)
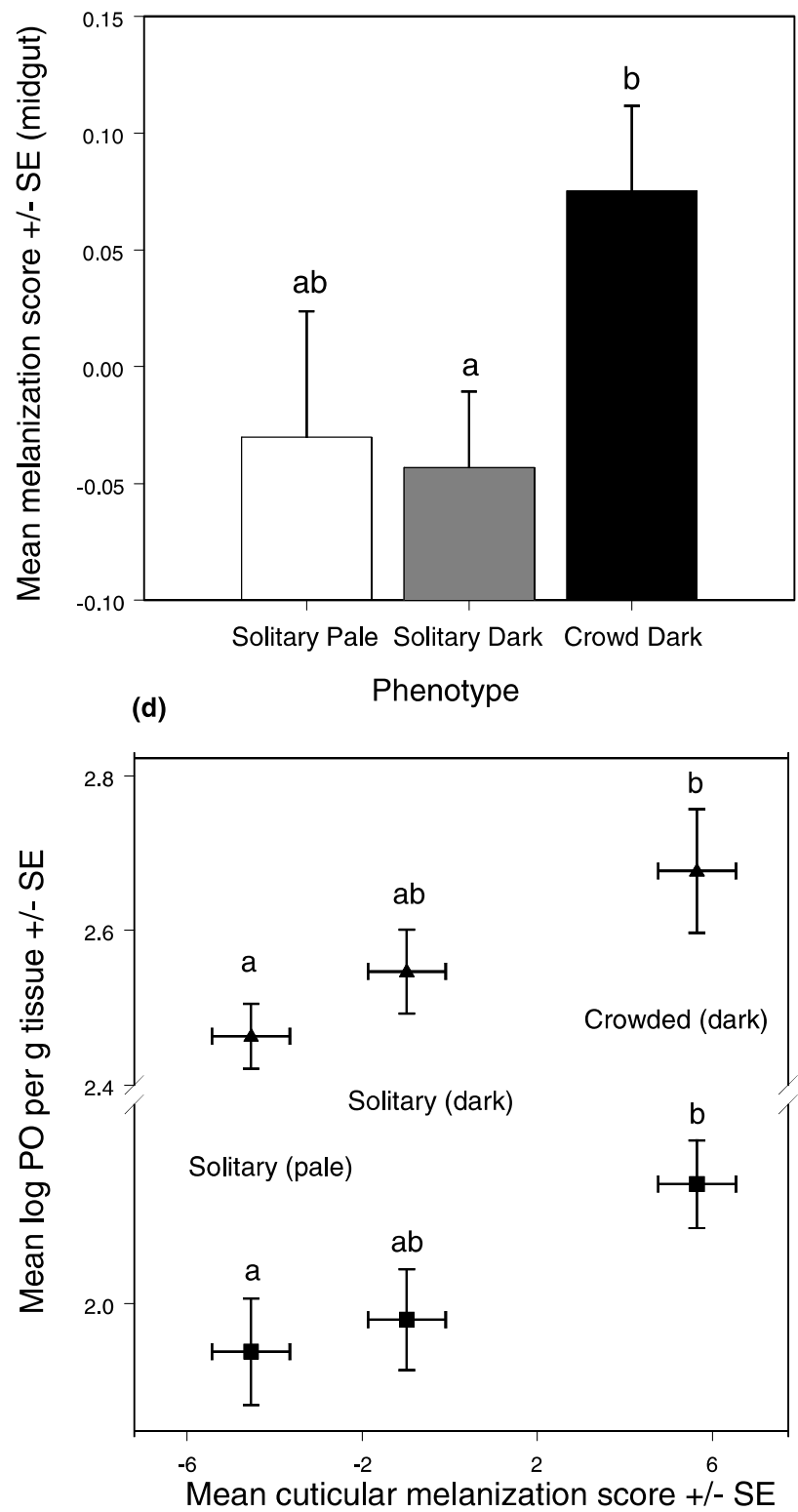

Figure 2 Relationship between larval phenotype and (a, b) melanization scores and (c, d) phenoloxidase activity. In the top panel, the maximum melanization score (mean \pm SE) is plotted against larval phenotype (solitary-pale, solitary-dark, crowded-dark) for (a) the cuticle and (b) the midgut. In the bottom panel, PO activity in (c) the haemolymph and (d) the cuticle and midgut is plotted against mean melanization score for each larval phenotype. In (c) PO activity (mean \pm standard error) is expressed as PO units per mg protein. Symbols sharing a common superscript are not significantly different from each other, based on Fisher's LSD multiple comparisons following onefactor ANOVAs with larval phenotype as the factor. In (d), this applies only within tissue-type; comparisons across tissue types are not valid. Correlations across phenotypes and melanization scores are shown in Table 1.

$P>0.5$; maximum: $F_{1,50}=4.64, P=0.036$; Fig. $\left.2 \mathrm{~b}\right)$ was largely a consequence of density-dependent differences in the maximum melanization score.

Analyses of whole mounts of cuticles under a light microscope showed that variation in the darkness of 'pale' and 'dark' caterpillars was due to variation in the density of small melanin granules (approx. $1 \mu \mathrm{m}$ diameter) in the dorso-lateral stripes (cf. Fig. 1b,c). Following incubation with L-dopa, all cuticles became much darker due to the uniform deposition of melanin (cf. Fig. 1b,d). 


\section{Larval density, colour and phenoloxidase activity}

Haemolymph PO activity increased with both rearing density (ANOVA using all larvae: $F_{1,58}=7.69, P=0.007$ ) and cuticle colour (ANOVA using solitary-reared larvae only: $F_{1,38}=10.86, P=0.002$ ), resulting in haemolymph PO activity being greatest in 'typical' (dark) crowded larvae, lowest in 'typical' (pale) solitary larvae and intermediate in dark-solitary larvae $\left(r_{\mathrm{s}}=0.651, \quad P<\right.$ 0.001; Fig. 2c). PO activity in both the cuticle and midgut also increased with increasing 'gregarization' from palesolitary to dark-crowded larvae (cuticle: $r_{\mathrm{s}}=0.312$, $P=0.016$; midgut: $r_{\mathrm{s}}=0.295, P=0.022$; Fig. $2 \mathrm{~d}$ ), due mainly to a significant difference between solitary and crowded larvae (cuticle: $F_{1,58}=6.38, P=0.014$; midgut: $\left.F_{1,58}=4.71, P=0.039\right)$, rather than between pale and dark solitary larvae (cuticle: $F_{1,38}=0.21, P>0.6$; midgut: $\left.F_{1,38}=0.84, P>0.3\right)$.

All three PO measurements were significantly positively correlated with each other; the average correlation across all three phenotypes was $r \geq 0.416, P \leq 0.001$ (Table 1). The partial correlation coefficients were also high and generally significantly different from zero (haemolymph PO - cuticle PO: $r_{\text {partial }}=0.354, P=0.006$; haemolymph PO - midgut $\quad$ PO: $\quad r_{\text {partial }}=0.255, \quad P=0.051 ; \quad$ cuticle PO - midgut PO: $\left.r_{\text {partial }}=0.330, P=0.010\right)$. Thus, it is appropriate to examine the relationship between phenoloxidase activity and other larval attributes using a multivariate approach.

There was a highly significant relationship between overall PO activity (in the haemolymph, cuticle and midgut) and larval rearing density (MANOva: Wilks' $\Lambda=0.719$, $\left.F_{3,56}=7.29, P<0.001\right)$, with all three of these measurements making significant, independent contributions to the strength of the relationship (univariate tests: haemolymph PO: $F_{1,58}=21.91, P<0.001$; cuticle PO: $F_{1,58}=6.39$, $P=0.014$; midgut PO: $\left.F_{1,58}=4.72, P=0.034\right)$. Similar results were obtained when larval phenotype (1-3) was the predictor variable (Wilks' $\Lambda=0.616, \quad F_{3,56}=11.63$, $P<0.001$; univariate tests: haemolymph PO: $F_{1,58}=$ 35.90, $P<0.001$; cuticle PO: $F_{1,58}=5.81, \quad P=0.019$; midgut PO: $F_{1,58}=5.55, P=0.022$ ). When colour (pale or dark) was the predictor variable (for solitary larvae), the MANOVA remained statistically significant, but this was due primarily to the association between colour and haemolymph PO (Wilks' $\Lambda=0.767, F_{3,36}=3.63, \quad P=0.022$; univariate tests: haemolymph PO: $F_{1,38}=10.86, \quad P=$ 0.002 ; cuticle PO: $F_{1,38}=0.19, P>0.6$; midgut PO: $F_{1,38}=1.54, P>0.2$ ). It is unclear at this stage whether the non-significance of the two other PO measures is due to genuine biological reasons or whether it is simply a consequence of the relatively small number of solitary larvae examined.

\section{Phenoloxidase activity and melanization of the cuticle and midgut}

PO activity in all three tissues (haemolymph, midgut and cuticle) was significantly positively correlated with one or both of the cuticular melanization measurements (Table 1). When all three PO measurements were included as dependent variables in a multivariate ANOVA, there was a significant positive relationship between the cuticular melanization score and overall PO activity (MANOva: Wilks' $\Lambda=0.839, F_{3,56}=3.59, P=0.019$; univariate tests: haemolymph PO: $F_{1,58}=9.27, \quad P=0.004$; cuticle PO: $F_{1,58}=5.38, \quad P=0.024 ; \quad$ midgut $\quad$ PO: $\quad F_{1,58}=4.39$, $P=0.040)$. The fact that all three of the univariate tests were independently statistically significant indicates that cuticular melanization is a good indicator of relative investment in PO activity in all three tissues (Figs 2c,d).

The degree of midgut melanization was significantly positively correlated with haemolymph PO $(r=0.292$, $n=60, P=0.037)$, but not with midgut PO $(r \leq 0.156$, $P \geq 0.265)$ or cuticular melanization score $(r \leq 0.214$, $P \geq 0.127$ ). When all three phenoloxidase measurements were included as dependent variables in a MANOVA, the association between PO activity and midgut melanization was non-significant (MANOVA: Wilks' $\Lambda=0.888, F_{3,48}=$ 2.01, $P=0.125)$.

\section{Resistance to ectoparasitoids}

The number of eggs laid by ovipositing female E. laphygmae ranged between 1 and 15, and was independent of larval colour and rearing density (linear regression: $F_{1,87}<1.28$, $P>0.25)$. The proportion of eggs that became melanized was independent of the number of eggs laid on each host (logistic regression: $\chi_{1}^{2}=0.083, P>0.77$ ) and the density at which the host was reared $\left(\chi_{1}^{2}=0.093, P>0.77\right)$. However, there was a strong positive relationship between larval colour and the proportion of eggs that became melanized $\left(\chi_{1}^{2}=9.003, P=0.003\right.$; Fig. 3). In other words, the darker the cuticle, the greater the proportion of parasitoid eggs that became melanized.

\section{Resistance to entomopathogenic fungi}

Fungus-induced mortality in $S$. littoralis was significantly affected by dose (logistic regression: $\chi_{1}^{2}=7.86, P=0.005$; Figs 4a,b), larval colour $\left(\chi_{1}^{2}=6.370, P=0.012\right.$; Fig. 4a) and rearing density $\left(\chi_{1}^{2}=4.569, P=0.033\right.$; Fig. $\left.4 \mathrm{~b}\right)$. None of the interaction terms were statistically significant. Thus, at both doses, fungus-induced mortality in $S$. littoralis was significantly greater in solitary-reared caterpillars and in caterpillars with pale cuticles than in crowd-reared caterpillars and those with dark cuticles, and these effects were 


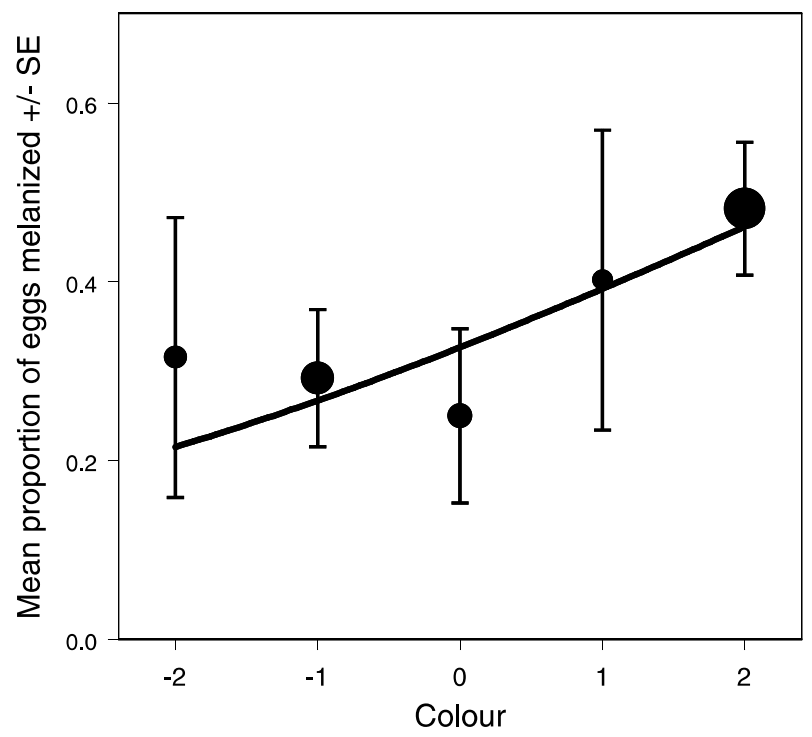

Figure 3 Relationship between cuticular melanization and resistance to the ectoparasitoid E. laphygmae. The vertical axis shows the proportion of melanized eggs $( \pm \mathrm{SE})$ as a function of degree of cuticular melanization, scored on a scale from -2 (very pale) to +2 (very dark). Symbol size reflects sample size. The line is the fitted logistic regression to the raw data.

additive. There was no fungus-induced mortality in the control group.

Fungus-induced mortality was much greater for $S$. exempta than S. littoralis (Fig. 4). There was a significant effect of dose on mortality in $S$. exempta $\left(\chi_{1}^{2}=24.81, P<0.001\right)$. Although rearing density $\left(\chi_{1}^{2}=0.001, \mathrm{~ns}\right)$ and colour $\left(\chi_{1}^{2}=0.43\right.$, ns) were non-significant as main effects, there were significant interactions between dose and rearing density $\left(\chi_{1}^{2}=13.72, P<0.001\right.$; Fig. $\left.4 \mathrm{a}\right)$ and between rearing density and colour $\left(\chi_{1}^{2}=5.47, P<0.02\right)$. When the lowand high-dose treatments were analysed separately, fungusinduced mortality was significantly greater in solitary larvae than crowded larvae in the low-dose treatment $(64 \%$ vs. $\left.44 \% ; \chi_{1}^{2}=5.63, P=0.017\right)$, whereas the trend was significant and reversed in the high-dose treatment $(73 \%$ vs. $89 \% ; \chi_{1}^{2}=7.03, P=0.008$; Fig. 4a).

\section{DISCUSSION}

Our results provide further support for an association between population density, melanism and disease resistance in insects. Image-analysis indicated that there are three quantifiable phenotypes of $S$. exempta, which can be considered to lie on a gregarization scale, with pale-solitary larvae the least 'gregarized' and dark-crowded larvae the most, whereas dark-solitary larvae are at an intermediate position. This is in accord with our earlier observation that dark-solitary larvae show levels of resistance to a baculovirus (a)

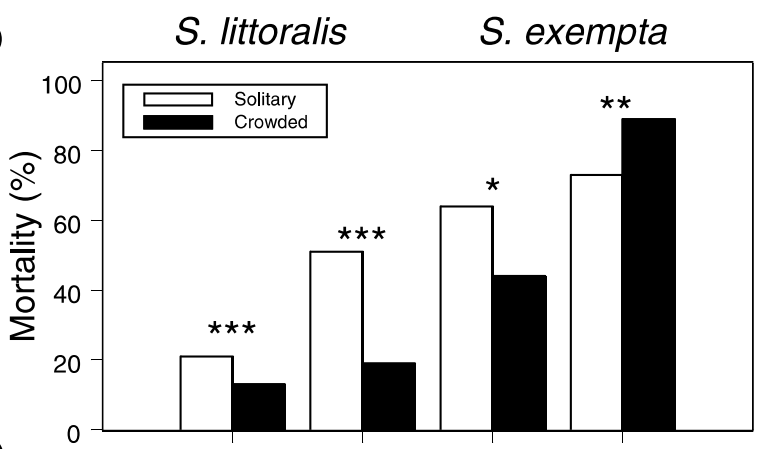

(b)

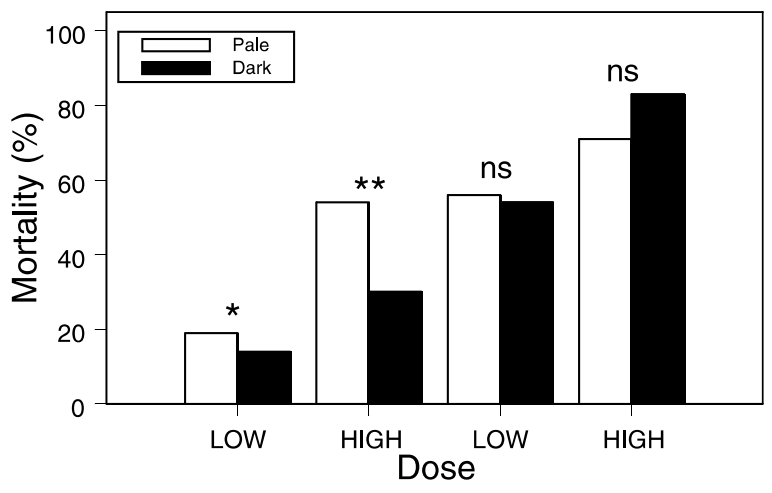

Figure 4 Resistance to B. bassiana in relation to (a) rearing density and (b) cuticular melanization. In (a) the comparison is between larvae reared solitarily and those in crowds (three or four larvae per pot); in (b) the comparison is between larvae with pale cuticles and those with dark cuticles. Low dose refers to $1 \times 10^{8}$ conidia $\mathrm{mL}^{-1}$ and $1 \times 10^{9}$ conidia $\mathrm{mL}^{-1}$. Symbols above the bars refer to the statistical significance of the difference between treatments, as determined by logistic regression: ns $P>0.05, * P<0.05$, ${ }^{* *} P<0.01,{ }^{* * *} P<0.001$. See text for details of full analysis.

that are intermediate between pale-solitary and darkcrowded larvae (Reeson et al. 1998). An association between melanism and rearing density was also observed in the midgut, suggesting that melanin in the midgut might also be functional and aid in resisting pathogens, such as baculoviruses, that enter the host orally (see below). Overall, the midgut showed much lower levels of melanization than the cuticle, despite having higher levels of $\mathrm{PO}$ activity. This may reflect differences in the availability of PO substrates, as melanization in the midgut is likely to be constrained by functional requirements for the exchange of nutrients.

The density-dependent prophylaxis (DDP) hypothesis proposes that if the risk of being exposed to pathogens increases with density due to density-dependent pathogen transmission, then insects will use density as a cue to match their levels of investment in immune function to the perceived risk (Wilson \& Reeson 1998). Consistent with this hypothesis, we found that PO levels in the haemolymph, cuticle and midgut all, independently, increased with 
increasing 'gregarization'. Thus, the lowest PO levels were observed in pale, solitary-reared caterpillars and the highest were in dark, crowd-reared caterpillars. Within solitaryreared caterpillars, PO activity in all three tissues was greater in dark individuals than pale, although the difference was significant only for PO in the haemolymph. Thus, independent of rearing density, melanism tended to be associated with elevated PO activity, particularly in the haemolymph.

The positive correlations (and partial correlations) between PO activity in the haemolymph, cuticle and midgut indicate that individuals investing in pathogen resistance do so at all sites where pathogen defence might be required; there is no evidence from these data for a phenotypic tradeoff between levels of PO expression at the different sites (although this does not mean that one does not exist; Lessells 1991). Siva-Jothy et al. (2001) present evidence suggesting that maintaining high PO activity in both the haemolymph and midgut is costly; however, gregarized $S$. exempta larvae appear to be able to pay such costs, at least in the absence of an immune challenge.

The increase in midgut PO levels with increasing gregarization is particularly interesting because the area around the midgut is one of the main sites of resistance to pathogens that infect orally. Therefore, we might expect that if $\mathrm{PO}$ is involved in this process then elevated midgut $\mathrm{PO}$ levels would be associated with increased resistance to such pathogens. Although it is not possible to test this hypothesis directly, it is suggestive that (across phenotypes) resistance to nucleopolyhedrovirus (NPV) in S. exempta was positively correlated with mean PO activity in the midgut (Fig. 5; NPV

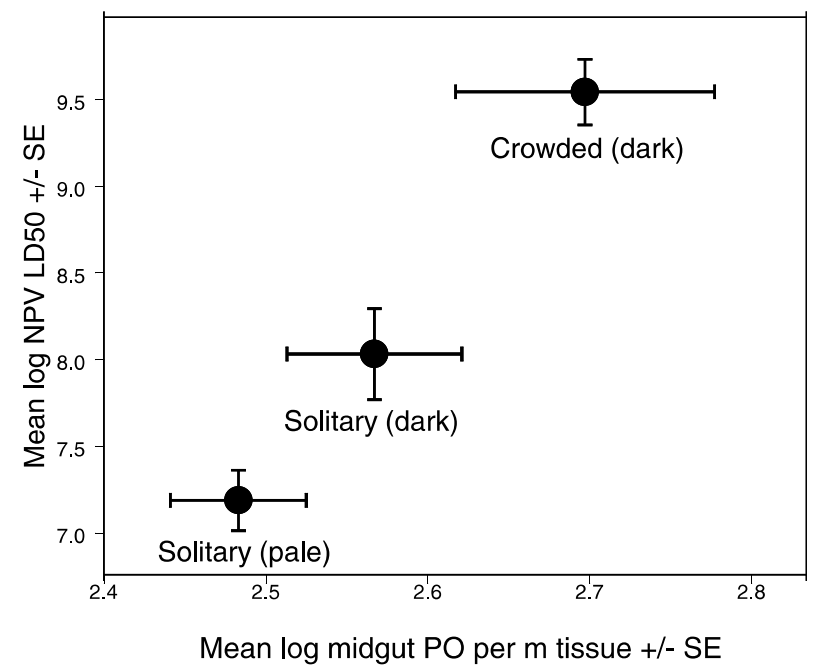

Figure 5 Relationship between midgut phenoloxidase levels and resistance to nuclear polyhedrosis virus. Mean $\mathrm{LD}_{50}$ data for S. exempta NPV come from Reeson et al. (1998), midgut phenoloxidase levels are from the present study. bioassay data from Reeson et al. 1998). However, this result should be viewed with some caution because resistance to baculoviruses occurs within the haemolymph as well as the midgut and it is possible that the correlation between phenotype and NPV resistance is associated with variation in haemolymph PO activity rather than PO activity in the midgut (or indeed that it is generated by an alternative, as yet unidentified, mechanism).

If dark cuticles have higher levels of PO, then it is reasonable to assume that they will be better able to resist parasites and pathogens that invade their hosts via the cuticle. Results from the parasitoid experiment suggest that dark larvae are significantly better at melanizing ectoparasitoid eggs attached to the cuticle (Fig. 3), so preventing them from developing further. High PO activity in the haemolymph is associated with greater capacity to melanize and/or encapsulate foreign objects, including parasites and parasitoids, that enter the haemocoel (Rizki \& Rizki 1990; Nappi et al. 1992; Shiao et al. 2001; Cotter \& Wilson 2001). Due to the covariation between melanism and PO activity in the cuticle and haemolymph, melanic larvae are likely to be more resistant to both ecto- and endo-parasitoids. This leads to the intriguing possibility that the melanin in the cuticle might act as an honest signal to parasitoids of an immunocompetent host that has high PO activity and should be avoided. There is some evidence that parasitoids can distinguish between different colour morphs of aphids and preferentially oviposit in the morph with the lower physiological resistance (e.g. Ankersmit et al. 1981, 1986; Michaud \& Mackauer 1994, 1995; Battaglia et al. 1995), and Verhoog et al. (1996) found that the parasitoid wasp Venturia canescens was 'not eager' to parasitize a melanic strain of the Mediterranean mealmoth, Ephestia kuehniella, and its ovipositor 'sometimes appeared to become stuck in the cuticle' (suggesting that the melanized cuticle may also provide a tougher physical barrier). We found no evidence that E. laphygmae altered its clutch size in relation to $S$. exempta melanism. However, our experimental design did not allow the parasitoid to choose between melanic and non-melanic larvae and so may not be an appropriate test of this hypothesis.

There is evidence from calopterygid damselflies that melanin might signal immunocompetence, not to parasitoids, but to potential mates. Calopteryx splendens is a sexually dimorphic damselfly and males have prominent wing patches that contain melanin. Variation in the wing patch morphology affects the outcome of both intrasexual interactions and female reproductive decisions after courtship (Siva-Jothy 2000). Rantala et al. (2000) found that males with larger wing patches were better able to encapsulate an artificial parasite (a small piece of nylon monofilament), and Siva-Jothy (2000) found that males with wing patches that were more homogeneous (and presumably contained more 
melanin) had lower burdens of a eugregarine parasite. Given the apparent importance of wing patches in inter- and intrasexual interactions, it seems likely that melanin is involved in signalling some aspect of male condition or immunocompetence in this species.

Insect colour is more commonly associated with defence against predators, and it has been suggested that densitydependent colour change might have evolved in insects as a density-dependent antipredator strategy: crypsis at low densities and aposematism at high densities (Sword 1999; Sword et al. 2000). Indeed, recently it has been speculated that this hypothesis might be applicable to those Lepidopteran larvae (including Spodoptera species) that exhibit densitydependent melanism (Wilson 2000). However, as yet, there is no evidence in support of this assertion, although experiments aimed at testing this hypothesis are underway (K. Wilson, unpublished).

The results from the fungus experiments suggest different patterns for the two Spodoptera species. As predicted by the DDP hypothesis, crowded larvae were significantly more resistant to fungal infection than solitary larvae at high and low doses for S. littoralis and at low doses for S. exempta (Fig. 4a). However, at the high dose, crowded S. exempta larvae were more susceptible to the fungus, suggesting that there might be a trade-off that results in greater resistance of crowded larvae when pathogen density is below some threshold, but lower resistance when pathogen density is above it. This might occur if, for example, crowded $S$. exempta larvae invest more in cuticular defences at the expense of haemolymph defences. Such a trade-off would be generated if a limiting resource for immune defence (e.g. PO substrate) is irreversibly allocated to one or other tissue (see Siva-Jothy et al. 2001), or if a limiting resource (e.g. an amino acid) could be used either to manufacture a biochemical that is important in cuticular defence (e.g. melanin) or to manufacture a different biochemical that aids in haemolymph defence (e.g. an antifungal protein). Either way, individuals investing in cuticular defences would do so at a cost to haemolymph defences and could expose themselves to increased risk at high spore densities, when the cuticle is likely to be penetrated. This is analogous to the dilemma facing an army defending a fort: putting all of ones soldiers on the perimeter fences may work well if opposition numbers are low, but if they are high, then as soon as these defences are breached, the battle will be lost.

An alternative explanation is that, at high fungal doses, numerous fungal spores could penetrate the host simultaneously, causing premature insect death (e.g. due to water loss, etc.) before the fungus could effectively exploit the resource. This would result in some larvae dying due to fungal invasion very rapidly after inoculation, but without subsequent sporulation on the cadaver. In support of this idea, we found that when we re-analysed the fungus-bioassay data for $S$. exempta and included all deaths, including those where there was no obvious mycosis, the interaction between fungal dose and rearing density became marginally non-significant $\left(\chi_{1}^{2}=3.66\right.$, $P=0.056$ ).

The relationship between colour and fungus-induced mortality was similar to that observed with respect to larval density. As predicted, dark larvae were better than pale larvae at resisting fungal infection at both low and high doses for $S$. littoralis and at low doses for $S$. exempta. But, again, this general trend was reversed for $S$. exempta at the high dose, although the difference between the colour morphs was non-significant at both doses (Fig. 4b). Thus, it appears that the different patterns for the two species may be a consequence of their relative susceptibilities to this strain of fungus: larval mortality in S. littoralis was just $16 \%$ and $41 \%$ for the low and high doses, respectively, whereas they were $54 \%$ and $79 \%$ for $S$. exempta. Similar patterns may have been produced for the two species had lower doses been used for $S$. exempta.

The results for $S$. littoralis are similar to those observed for the mealworm beetle T. molitor infected with the entomopathogenic fungus Metarbizium anisopliae (Barnes \& SivaJothy 2000), and are also in accord with those for the armyworm $M$. separata percutaneously infected with the entomopathogenic fungus Nomuraea rileyi (Mitsui \& Kunimi 1988). Mitsui \& Kunimi (1988) found that solitary-reared larvae were more susceptible to the fungus than crowdreared larvae $\left(\mathrm{LC}_{50} \mathrm{~s}\right.$ : solitary $=11.5 \times 10^{7}$, crowded $=$ $25.1 \times 10^{7}$ ), and that when larvae were reared gregariously, pale individuals were substantially more susceptible than dark ones $\left(\mathrm{LC}_{50}\right.$ s: pale $=4.9 \times 10^{7}$, dark $\left.=27.2 \times 10^{7}\right)$.

Our results suggest that there are several related properties of the DDP phenomenon that are likely to yield the observed positive association between melanism and disease resistance. First, because of the structural properties of melanin, a melanized cuticle is likely to provide a more secure physical barrier to the penetration of cutaneously entering parasites and pathogens. Second, because of the chemical properties of melanin, and in particular its inhibitory effect on lytic enzymes (Kuo \& Alexander 1967; Bull 1970), the ability of organisms to penetrate and grow on melanized cuticles may be reduced (e.g. St. Leger et al. (1988)). Third, as shown in the present study, melanized cuticles have greater PO activity, which can lead to the production of melanin in response to appropriate cues, including those produced by cuticle-penetrating fungal hyphae and by parasitoid eggs attached to the cuticle. Although our studies did not establish whether insects that resisted fungal attack had a stronger encapsulation response to invading fungi, they have shown that melanic larvae have a stronger melanization reaction to attached parasitoid eggs, 
a reaction that is catalysed by enzymes of the prophenoloxidase cascade.

In conclusion, we have shown that an increase in larval density stimulates increased production of melanin in both the cuticle and, to a lesser extent, the midgut; an act that is likely to enhance larval immunocompetence (sensu Owens \& Wilson 1999) as a consequence of the physical and chemical properties of melanin. We have also shown that melanism is associated with elevated PO activity, not only in the haemolymph but also at two of the barriers to pathogen penetration, the cuticle and the midgut. High levels of cuticular PO are associated with increased resistance to an ectoparasitic wasp and an entomopathogenic fungus; high levels of midgut PO are correlated with increased resistance to a baculovirus. These results suggest that melanism is a reliable indicator of $\mathrm{PO}$ production in the cuticle and midgut, and of resistance to parasites and pathogens that enter their host percutaneously or orally. Given recent studies indicating a potential role for melanin in the innate immune defences of vertebrates, including man (Mackintosh 2001), the present study suggests that the functional significance of melanism may have been underestimated.

\section{ACKNOWLEDGEMENTS}

This work was conducted whilst K.W. was in receipt of a Natural Environment Research Council, UK, Advanced Research Fellowship. J.K.P. is funded by the Ministry of Agriculture Fisheries and Food, U.K. IACR-Rothamsted receives grant-aided support from the Biotechnology and Biological Sciences Research Council, U.K. The purchase of the image-analysis suite was supported by a grant to K.W. from The Royal Society. We thank Andy Barnes, Tim Benton, Tim Carty, Jenny Cory, Alan Gunn, Rosie Hails, Mike Siva-Jothy, Sue Smith, Tim Whalley and Helen Yeo for their practical assistance and discussion of some of the ideas raised in this paper. We also thank Charles Dewhurst, Mark Parnell and David Gryzwacz (Natural Resources Institute, UK), Wilfred Mushobozi, Esther Kilonzo and the late Eugene Masawe (Pest Control Services, Arusha, Tanzania) for their assistance in the collection of field samples, and four anonymous referees for making suggestions that greatly improved the paper.

\section{REFERENCES}

Ankersmit, G.W., Acreman, T.M. \& Dijkman, H. (1981). Parasitism of color forms in Sitobion avenae. Entomologia Expis Applicata, 29, 362-363.

Ankersmit, G.W., Bell, C., Dijkman, H., Mace, N., Riestra, S., Shröder, J. \& de Visser, C. (1986). Incidence of parasitism by Aphidius rhopalosiphi. colour forms of the aphid Sitobion avenae. Entomologia Expis Applicata, 40, 223-229.
Ashida, M. \& Söderhäll, K. (1984). The prophenoloxidase activating system in crayfish. Comp. Biochem. Physiol. B-Biochem. Mol. Biol., 77, 21-26.

Barnes, A.I. \& Siva-Jothy, M.T. (2000). Density-dependent prophylaxis in the mealworm beetle Tenebrio molitor L-(Coleoptera: Tenebrionidae): cuticular melanization is an indicator of investment in immunity. Proc. Roy. Soc. Lond. Series B - Biol. Sci., 267, 177-182.

Battaglia, D., Pennacchio, F., Romano, A. \& Tranfaglia, A. (1995). The role of physical cues in the regulation of host recognition and acceptance behavior of Aphidius ervi Haliday (Hymenoptera, Braconidae). J. Insect Behav., 8, 739-750.

Beckage, N., Thompson, S. \& Federici, B., eds. (1993). Parasites and Pathogens of Insects. Academic Press, Inc, San Diego.

Bull., A. (1970). Inhibition of polysaccharases by melanin: enzyme inhibition in relation to mycolysis. Arch. Biochem. Biophysics, 137, 345-356.

Collins, F.H., Sakai, R.K., Vernick, K.D., Paskewitz, S., Seeley, D.C., Miller, L.H., Collins, W.E., Campbell, C.C. \& Gwadz, R.W. (1986). Genetic selection of a Plasmodium-refractory strain of the malaria vector Anopheles gambiae. Science, 234, 607-610.

Cotter, S.C. \& Wilson, K. (2001) Hertability of immune function in the caterpillar Spodoptera littoralis. Heredity, in press.

Doering, T.L., Nosanchuk, J.D., Roberts, W.K. \& Casadevall, A. (1999). Melanin as a potential cryptococcal defence against microbicidal proteins. Med. Mycol., 37, 175-181.

Faure, J. (1943). Phase variation in the armyworm Laphygma exempta (Walk,). Scientific Bulletin of the Department of Agriculture and Forestry of the Union of South Africa, 17.

Götz, P. (1986). Encapsulation in arthropods. In: Immunity in Invertebrates (ed. M. Brehelin). Springer, Berlin, pp. 153-170.

Goulson, D. (1994). Determination of larval melanization in the moth, Mamestra brassicae, and the role of melanin in thermoregulation. Heredity, 73, 471-479.

Goulson, D. \& Cory, J.S. (1995). Responses of Mamestra brassicae (Lepidoptera, Noctuidae) to crowding - interactions with disease resistance, color phase and growth. Oecologia, 104, 416-423.

Gunn, A. (1998). The determination of larval phase coloration in the African armyworm, Spodoptera exempta and its consequences for thermoregulation and protection from UV light. Entomologia Expis Applicata, 86, 125-133.

Hajek, A.E. \& St. Leger, R.J. (1994). Interactions between fungal pathogens and insect hosts. Annu. Rev. Entomol., 39, 293-322.

Hiruma, K. \& Riddiford, L.M. (1988). Granular phenoloxidase involved in cuticular melanization in the tobacco hornworm regulation of its synthesis in the epidermis by juvenile-hormone. Dev. Biol., 130, 87-97.

Hodjat, S. (1970). Effects of crowding on colour, size and larval activity of Spodoptera littoralis (Lepidoptera: Noctuidae). Entomologia Expis Applicata, 13, 97-106.

Hung, S.Y. \& Boucias, D.G. (1992). Influence of Beauveria bassiana on the cellular defense response of the beet armyworm, Spodoptera exigua. J. Invertebrate Pathol., 60, 152-158.

Ishikawa, H., Mitsui, Y., Yoshitomi, T., Mashimo, K., Aoki, S., Mukuno, K. \& Shimizu, K. (2000). Presynaptic effects of botulinum toxin type A on the neutrally evoked response of albino and pigmented rabbit iris sphincter and dilator muscles. Jap. J. Opthalmol., 44, 106-109.

Kazimirova, M. (1992). The role of physical contact in the induction of phase polymorphism of Mamestra brassicae (Lepidoptera, Noctuidae). Acta Entomologica Bohemoslovaca, 89, 87-95. 
Kunimi, Y. \& Yamada, E. (1990). Relationship of larval phase and susceptibility of the armyworm, Psendaletia separata walker (Lepidoptera, Noctuidae) to a nuclear polyhedrosis-virus and a granulosis-virus. Appl. Entomol. Zool., 25, 289-297.

Kuo, M. \& Alexander, M. (1967). Inhibition of the lysis of fungi by melanins. J. Bacteriol., 94, 624-629.

Lessells, C. (1991). The evolution of life histories. In: Behavioural Ecology: an Evolutionary Approach (eds J. Krebs \& N. Davies). Blackwell Scientific Publications, Oxford, pp. 482.

Long, D. (1953). Effects of population density on larvae of Lepidoptera. Trans. Roy. Entomol. Soc. London, 104, 543-585.

Mackintosh, J.A. (2001). The antimicrobial properties of melanocytes, melanosomes and melanin and the evolution of black skin. J. Theoret. Biol., 211, 101-113.

Majerus, M. (1998). Melanism: Evolution in Action. Oxford University Press, Oxford.

Marmaras, V.J., Bournazos, S.N., Katsoris, P.G. \& Lambropoulou, M. (1993). Defense-mechanisms in insects - certain integumental proteins and tyrosinase are responsible for nonselfrecognition and immobilization of Escherichia coli. the cuticle of developing Ceratitis capitata. Arch. Insect Biochem. Physiol., 23, 169-180.

Mason, H. (1955). Comparative biochemistry of the phenolase comples. Adv. Ensymol., 16, 105-184.

Media Cybernetics (1999). Image-Pro Plus. Media Cybernetics, Silver Spring, MD.

Michaud, J.P. \& Mackauer, M. (1994). The use of visual cues in host evaluation by aphidiid wasps 1 . Comparison between 3 Aphidius parasitoids of the pea aphid. Entomologia Expis Applicata, 70, 273-283.

Michaud, J.P. \& Mackauer, M. (1995). The use of visual cues in host evaluation by aphidiid wasps 2. Comparison between Ephedrus californicus. Monoctonus paulensis, and Praon pequodorum. Entomologia Expis Applicata, 74, 267-275.

Mitsui, J. \& Kunimi, Y. (1988). Effect of larval phase on susceptibility of the armyworm, Pseudaletia separata Walker (Lepidoptera: Noctuidae) to an entomogeneous deuteromycete, Nomuraea rileyi. Jap. J. Appl. Entomol. Zoology, 32, 129-134.

Montefiori, D.C. \& Zhou, J. (1991). Selective antiviral activity of synthetic soluble L-tyrosine and L-Dopa melanins against human immunodeficiency virus in vitro. Antiviral Res., 15, 11-26.

Nappi, A.J., Carton, Y., Li, J. \& Vass, E. (1992). Reduced cellular immune competence of a temperature-sensitive dopa decarboxylase mutant strain of Drosophila melanogaster against the parasite Leptopilina boulardi. Comp. Biochem. Physiol. B, 101, 453-460.

Nappi, A.J. \& Vass, E. (1993). Melanogenesis and the generation of cytotoxic molecules during insect cellular immune-reactions. Pigment Cell Res., 6, 117-126.

Nigam, Y., Maudlin, L., Welburn, S. \& Ratcliffe, N. (1997). Detection of phenoloxidase activity in the hemolymph of tsetse flies, refractory and susceptible to infection with Trypanosoma brucei rhodensie. J. Invertebrate Pathol., 69, 279-281.

Ourth, D.D. \& Renis, H.E. (1993). Antiviral melanization reaction of Heliothis virescens against DNA and RNA viruses in vitro. Comp. Biochem. Physiol., 105B, 719-723.

Owens, I.P.F. \& Wilson, K. (1999). Immunocompetence: a neglected life history trait or conspicuous red herring? Trends Ecol. Evol., 14, 170-172.

Paskewitz, S.M., Brown, M.R., Collins, F.H. \& Lea, A.O. (1989). Ultrastructural-localization of phenoloxidase in the midgut of refractory Anopheles gambiae and association of the enzyme with encapsulated Plasmodium cynomolgi. J. Parasitol., 75, 594-600.

Paskewitz, S.M., Brown, M.R., Lea, A.O. \& Collins, F.H. (1988). Ultrastructure of the encapsulation of Plasmodium cynomolgi (b-strain) on the midgut of a refractory strain of Anopheles gambiae. J. Parasitol., 74, 432-439.

Poinar, G. (1974). Insect immunity to parasite nematodes. In: Contemporary Topics in Immunobiology, Vol. 4 (ed. E. Cooper). Plenum, New York, pp. 167-178.

Rantala, M.J., Koskimaki, J., Taskinen, J., Tynkkynen, K. \& Suhonen, J. (2000). Immunocompetence, developmental stability and wingspot size in the damselfly Calopteryx splendens L. Proc. Roy. Soc. Lond. Series B - Biol. Sci., 267, 2453-2457.

Reeson, A.F., Wilson, K., Cory, J.S., Hankard, P., Weeks, J.M., Goulson, D. \& Hails, R.S. (2000). Effects of phenotypic plasticity on pathogen transmission in the field in a LepidopteraNPV system. Oecologia, 124, 373-380.

Reeson, A.F., Wilson, K., Gunn, A., Hails, R.S. \& Goulson, D. (1998). Baculovirus resistance in the noctuid Spodoptera exempta is phenotypically plastic and responds to population density. Proc. Roy. Soc. Lond. Series B - Biol. Sci., 265, 1787-1791.

Rizki, R.M. \& Rizki, T.M. (1990). Encapsulation of parasitoid eggs in phenoloxidase-deficient mutants of Drosophila melanogaster. J. Insect Physiol., 36, 523-529.

Sambrook, J., Fritsch, E.F. \& Maniatis, T. (1989). Molecular Cloning: a Laboratory Manual, 2nd edn. Cold Spring Harbor Laboratory Press, New York.

Shiao, S.H., Higgs, S., Adelman, Z., Christensen, B.M., Liu, S.H. \& Chen, C.C. (2001). Effect of prophenoloxidase expression knockout on the melanization of microfilariae in the mosquito Armigeres sublabatus. Insect Mol. Biol., 10, 315-321.

Sidibe, S., Saal, F., RhodesFeuillette, A., Lagaye, S., Pelicano, L., Canivet, M., Peries, J. \& Dianoux, L. (1996). Effects of serotonin and melanin on in vitro HIV-1 infection. J. Biol. Regulators Homeostatic Agents, 10, 19-24.

Silva-Jothy, M.T., Tsubaki, Y., Hooper, R. \& Plaistow, S.J. (2001). Investment in immune function under chronic and acute immune challenge in an insect. Physiol. Entomol., 26, 1-5.

Siva-Jothy, M.T. (2000). A mechanistic link between parasite resistance and expression of a sexually selected trait in a damselfly. Proc. Roy. Soc. Lond. Series B - Biol. Sci., 267, 25232527.

Söderhäll, K. \& Ajaxon, R. (1982). Effect of quinones and melanin on mycelial growth of Aphanomyces spp and extracellular protease of Aphanomyces astaci a parasite on crayfish. J. Invertebrate Pathol., 39, 105-109.

St. Leger, R.J., Cooper, R.M. \& Charnley, A.K. (1988). The effect of melanization of Manduca sexta cuticle on growth and infection by Metarbizium anisopliae. J. Invertebrate Pathol., 52, 459-470.

St. Leger, R. (1991). Integument as a barrier to microbial infections. In: Physiology of the Insect Epidermis (eds K. Binnington \& A. Retnakaran). CSIRO, Australia, pp. 284-306.

Sword, G.A. (1999). Density dependent warning coloration. Nature, 397, 217-217.

Sword, G.A., Simpson, S.J., El Hadi, O.T.M. \& Wilps, H. (2000). Density dependent aposematism in desert locusts. Proc. Roy. Soc. Lond. Series B - Biol. Sci., 267, 63-68.

Verhoog, M., Boven, A. \& Brakefield, P. (1996). Melanic moths and the ability to encapsulate parasitoid eggs and larvae. Proc. Neth. Entomol. Soc., 7, 127-133. 
Washburn, J.O., Haas-Stapleton, E.J., Tan, F.F., Beckage, N.E. \& Volkman, L.E. (2000). Co-infection of Manduca sexta larvae with polydnavirus from Cotesia congregata increases susceptibility to fatal infection by Autographa californica M Nucleopolyhedrovirus. J. Insect Physiol., 46, 179-190.

Washburn, J.O., Kirkpatrick, B.A. \& Volkman, L.E. (1996). Insect protection against viruses. Nature, 383, 767-767.

Wilson, K. (2000). How the locust got its stripes: the evolution of density-dependent aposematism. Trends Ecol. Evol., 15, 88-90.

Wilson, K. \& Reeson, A.F. (1998). Density-dependent prophylaxis: evidence from Lepidoptera-baculovirus interactions? Ecol. Entomol., 23, 100-101.

Wolfgang, W.J. \& Riddiford, L.M. (1981). Cuticular morphogenesis during continuous growth of the final instar larva of a moth. Tissue Cell, 13, 757-772.

\section{BIOSKETCH}

Ken Wilson is an evolutionary ecologist interested in the impact of parasites on life-history evolution, and on the dynamics of host-parasite interactions. He works mainly on insects, especially Lepidopteran larvae, but also studies the behaviour, population dynamics and epidemiology of Soay sheep on St. Kilda, Scotland.

Editor, M. Hochberg

Manuscript received 14 September 2001

Manuscript accepted 17 September 2001 